\title{
Three points related to the incenter and excenters of a triangle
}

\begin{abstract}
Boris Odehnal
Boris Odehnal studierte von 1994 bis 1999 an der Technischen Universität Wien die Lehramtsfächer Mathematik und Darstellende Geometrie. Im Anschluß daran arbeitete er als Forschungsassistent am Institut für Geometrie der Technischen Universität Wien und gleichzeitig als Assistent an der Universität für Bodenkultur in Wien. Seit 2002 ist er am Institut für Diskrete Mathematik und Geometrie der Technischen Universität Wien tätig.
\end{abstract}

\section{Introduction}

Let $\Delta:=\{A, B, C\}$ be a triangle in the Euclidean plane. The side lengths of $\Delta$ shall be denoted by $c:=\overline{A B}, b:=\overline{A C}$ and $a:=\overline{B C}$. The interior angles enclosed by the edges of $\Delta$ are $\beta:=\angle A B C, \gamma:=\angle B C A$ and $\alpha:=\angle C A B$, see Fig. 1 .

It is well-known that the bisectors $w_{\alpha}, w_{\beta}$ and $w_{\gamma}$ of the interior angles of $\Delta$ are concurrent in the incenter $I$ of $\Delta$. The bisectors $\overline{w_{\beta}}$ and $\overline{w_{\gamma}}$ of the exterior angles at the vertices $A$ and $B$ and $w_{\alpha}$ are concurrent in the center $A_{1}$ of the excircle touching $\Delta$ along $B C$ from the outside.

Changing $\alpha, \beta$ and $\gamma$ cyclically, we can find the remaining two excenters $A_{2}$ and $A_{3}$. To get familiar with the notations used in this paper, see Fig. 1.

Here we remark that the base triangle $\Delta$ is the orthoptic triangle of the triangle built by the excenters. The orthocenter of $\Delta$ is the incenter of the orthoptic triangle. Later, when we give our theorems, a second interpretation will use this fact.

Die Inkreismitte und die drei Ankreismitten eines Dreiecks $\Delta$ sind die Mittelpunkte jener vier Kreise, die alle Seiten von $\Delta$ berühren. Aus diesen Punkten lassen sich drei weitere Punkte ableiten. Das so entstehende Dreieck $\Delta_{S}$ geht aus dem Dreieck $\Delta_{A}$ der Ankreismitten durch eine Halbdrehung um den FEUERBACH-Punkt von $\Delta$ hervor. Die Dreiecke $\Delta_{A}$ und $\Delta_{S}$ haben die EULER-Gerade und den Neunpunktekreis von FEUERBACH gemeinsam. In der vorliegenden Arbeit werden diese und weitere damit in Zusammenhang stehende Resultate mit elementaren Mitteln bewiesen. 

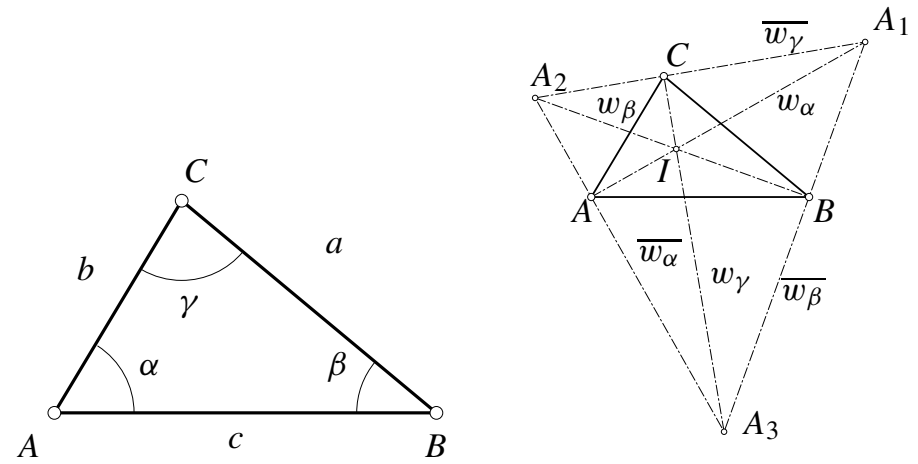

Fig. 1 Notations used in the paper

\section{Results and theorems}

Now we draw some normals emanating from the excenters $A_{i}$ and the incenter $I$. We use the symbol $n_{A_{1}}(A C)$ to indicate that this line is perpendicular to $A C$ and contains the point $A_{1}$. Drawing the lines $n_{A_{1}}(A C), n_{A_{2}}(B C)$ and $n_{I}(A B)$, respectively, we observe that these lines are concurrent in one point $S_{3}$. Cyclic rearrangement of $(A, B, C)$ and $(1,2,3)$ enables us to state the following theorem:

Theorem 2.1 The following triples of lines are concurrent:

(1) $\left(n_{A_{1}}(A C), n_{A_{2}}(B C), n_{I}(A B)\right)$ are concurrent in $S_{3}$.

(2) $\left(n_{A_{2}}(A B), n_{A_{3}}(A C), n_{I}(B C)\right)$ are concurrent in $S_{1}$.

(3) $\left(n_{A_{3}}(B C), n_{A_{1}}(A B), n_{I}(A C)\right)$ are concurrent in $S_{2}$.

Even in classical literature $[3,4]$ these points and the concurrencies of these normals are not mentioned. The concurrencies of the lines mentioned in Theorem 2.1 are illustrated in Fig. 2.

Moreover, we are able to prove the following result:

\section{Theorem 2.2}

(1) The circumcenter of the triangle $\Delta_{S}:=\left\{S_{1}, S_{2}, S_{3}\right\}$ is the incenter of $\Delta$.

(2) The circumradius of $\Delta_{S}$ equals twice the circumradius of $\Delta$.

For the sake of simplicity we use the abbreviation $\Delta_{A}:=\left\{A_{1}, A_{2}, A_{3}\right\}$ and state:

Theorem 2.3 The triangles $\Delta_{A}$ and $\Delta_{S}$ are congruent. There exists a rotation $\rho$ about the center of the FEUERBACH circle of $\Delta_{S}$ with angle $\phi=\pi$ with $\rho\left(\Delta_{A}\right)=\Delta_{S}$.

\section{Theorem 2.4}

(1) The FEUERBACH circle of $\Delta_{S}$ equals the circumcircle of $\Delta$.

(2) The triangles $\Delta_{A}$ and $\Delta_{S}$ share the FEUERBACH circle. 


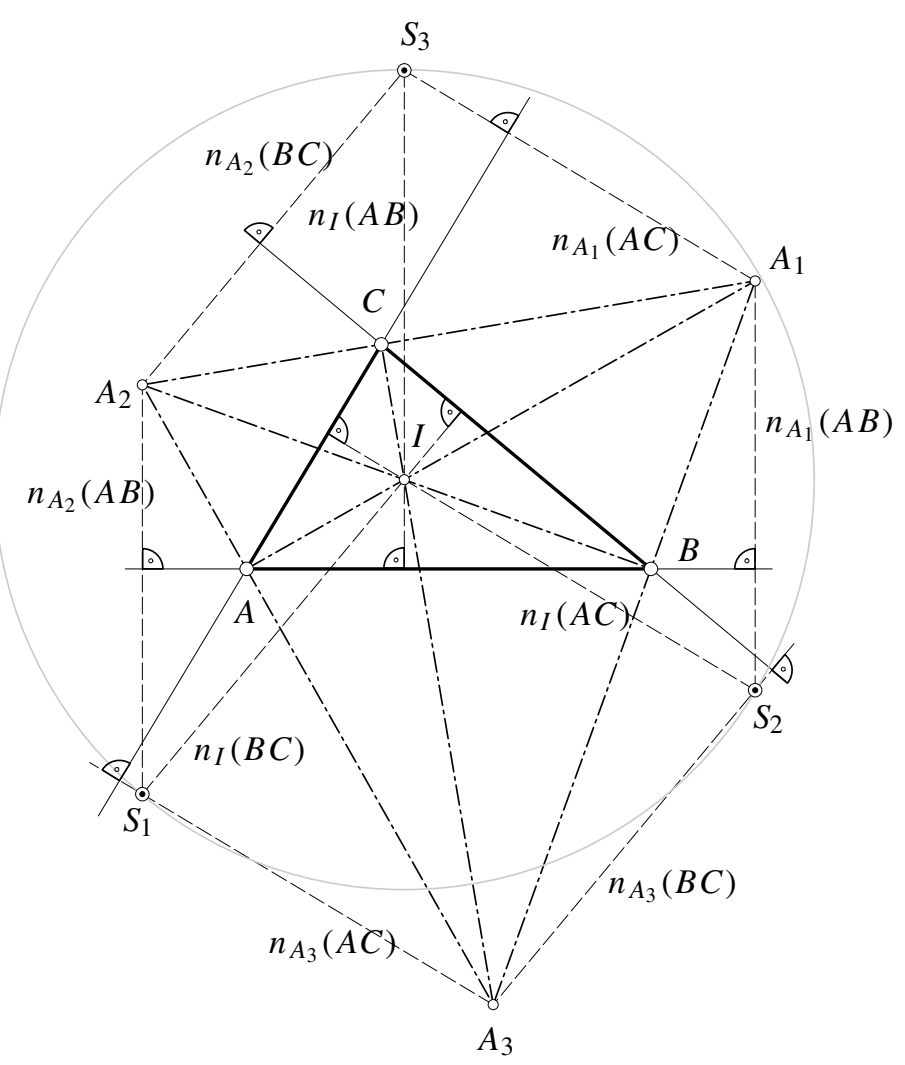

Fig. 2 Three remarkable points occuring as the intersection of some normals

\section{Theorem 2.5}

(1) The incenter I lies on the EULER line $e_{\Delta_{S}}$ of $\Delta_{S}$.

(2) The triangles $\Delta_{A}$ and $\Delta_{S}$ share the EULER line.

None of the above theorems are hitherto known. Even in $[3,4]$ the points $S_{i}$ and the theorems dealing with them are not mentioned.

\section{Proof of the main results}

Proof of Theorem 2.1. In order to show that the lines $n_{A_{1}}(A C), n_{A_{2}}(B C)$ and $n_{I}(A B)$ are concurrent in $S_{3}$, we compute the length of $I S_{3}$ in two different ways and obtain equal results. $\overline{I S_{3}}$ can be seen as the coordinate of the intersection points $n_{I}(A B) \wedge n_{A_{1}}(A C)$ and $n_{I}(A B) \wedge n_{A_{2}}(B C)$ on $n_{I}(A B)$.

We look at the triangles appearing in Fig. 3 and compute the length $\overline{I S_{3}}$. The first triangle to look at is $\Delta_{1}:=\left\{A, B, A_{1}\right\}$. The lengths of its edges are $\overline{A B}=c, \overline{B A_{1}}$ and $\overline{A_{1} A}$, 


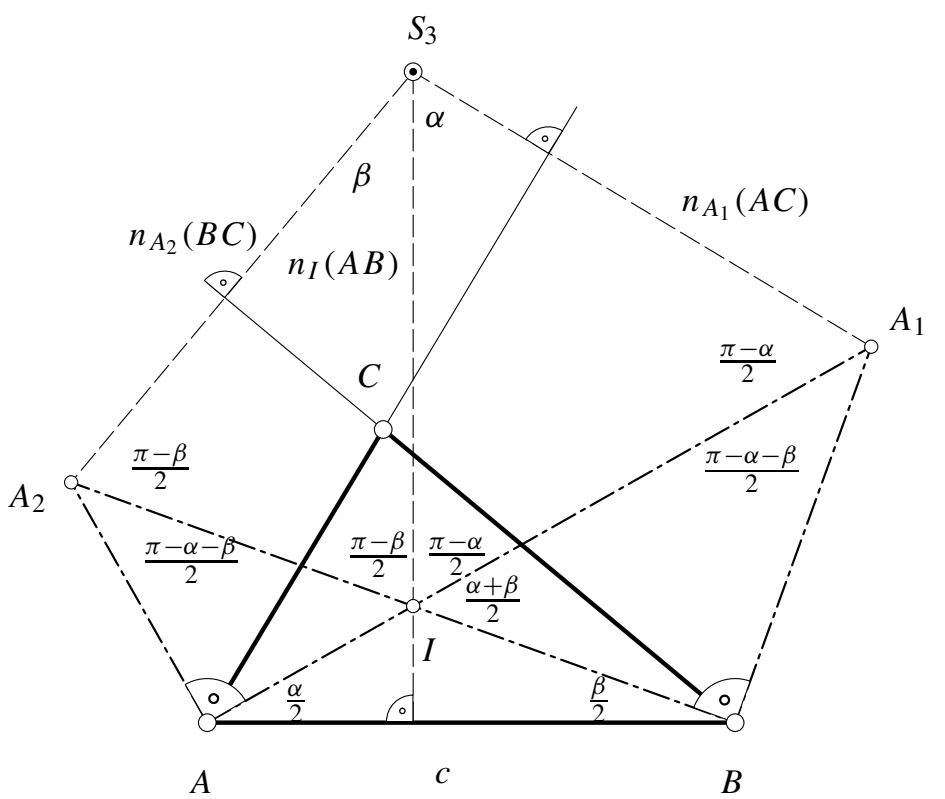

Fig. 3 Computation of $\overline{I S_{3}}$

respectively. The opposite angles have values $\frac{1}{2}(\pi-\alpha-\beta), \alpha / 2$ and $\frac{1}{2}(\pi+\beta)$. So we find

$$
\overline{A_{1} B}=c \frac{\sin \frac{\alpha}{2}}{\cos \frac{\alpha+\beta}{2}} .
$$

The next triangle we pay attention to is $\Delta_{2}:=\left\{B, I, A_{1}\right\}$. The lengths of edges appearing here are $\overline{B I}, \overline{I A_{1}}$ and $\overline{A_{1} B}$, respectively. The values of the angles lying opposite to these three edges are $\frac{1}{2}(\pi-\alpha-\beta), \pi / 2$ and $\frac{1}{2}(\alpha+\beta)$, respectively. Thus we find

$$
\overline{I A_{1}}=2 c \frac{\sin \frac{\alpha}{2}}{\sin (\alpha+\beta)} \text {. }
$$

At last we look at $\Delta_{3}:=\left\{I, A_{1}, S_{3}\right\}$ with edge lengths $\overline{I A_{1}}, \overline{A_{1} S_{3}}$ and $\overline{S_{3} I}$, respectively. The angles opposite to these edges have values $\alpha, \frac{1}{2}(\pi-\alpha)$ and $\frac{1}{2}(\pi-\alpha)$, respectively. Finally, we arrive at

$$
\overline{I S_{3}}=\frac{c}{\sin (\alpha+\beta)} .
$$

The computation of $\overline{I S_{3}}$ can be done in the same way with the triangles $\Delta_{1}^{\prime}:=\left\{A, B, A_{2}\right\}$, $\Delta_{2}^{\prime}:=\left\{A, I, A_{2}\right\}$ and $\Delta_{3}^{\prime}:=\left\{I, A_{2}, S_{3}\right\}$, which leads again to (3). Thus the coordinate of $n_{I}(A B) \wedge n_{A_{1}}(A C)$ and $n_{I}(A B) \wedge n_{A_{2}}(B C)$ on $n_{I}(A B)$ are equal and we have $n_{I}(A B) \wedge$ $n_{A_{1}}(A C)=n_{I}(A B) \wedge n_{A_{2}}(B C)=S_{3}$. 
Cyclic rearrangement of indices shows that the points $S_{1}$ and $S_{2}$ mentioned in Theorem 2.1 do exist and lie on the respective three normals.

We additionally obtain:

Theorem 3.1 The point $S_{i}$ is the circumcenter of the triangle $\left\{I, A_{j}, A_{k}\right\}$ and $(i, j, k)$ is either $(1,2,3)$ or $(2,3,1)$ or $(3,1,2)$.

Proof. Looking at triangles $\Delta_{4}:=\left\{I, A_{1}, S_{3}\right\}$ and $\Delta_{5}:=\left\{I, A_{2}, S_{3}\right\}$ we find

$$
\angle I A_{1} S_{3}=\angle A_{1} I S_{3} \text { and } \angle I A_{2} S_{3}=\angle A_{2} I S_{3} .
$$

So we have $\overline{I S_{3}}=\overline{A_{2} S_{3}}=\overline{A_{1} S_{3}}$. Rearranging the indices completes the proof.

Proof of Theorem 2.2. Replacing $(A, B, C)$ and $(1,2,3)$ cyclically in (3) we obtain

$$
\overline{I S_{1}}=\frac{a}{\sin (\beta+\gamma)} \text { and } \overline{I S_{2}}=\frac{b}{\sin (\alpha+\gamma)}
$$

Since the values of the interior angles sum up to $\pi$, that is $\gamma=\pi-\alpha-\beta$, we find

$$
\frac{c}{\sin (\alpha+\beta)}=\frac{c}{\sin \gamma}=2 R \text {. }
$$

Further, we use the well-known formulae

$$
\frac{c}{\sin \gamma}=\frac{b}{\sin \beta}=\frac{a}{\sin \alpha}=2 R,
$$

which gives a simple relation between the angles, the side lengths and the circumradius $R$ of $\Delta$. Thus the circumradius of $\left\{S_{1}, S_{2}, S_{3}\right\}$ is twice the circumradius of $\Delta$.

Proof of Theorem 2.3. In order to show that $\Delta_{A} \equiv \Delta_{S}$, we show that the lines $A_{1} A_{2}$ and $S_{1} S_{2}$ are parallel. (Equivalently, we could show that $A_{1} A_{3}$ and $S_{1} S_{3}$ are parallel and also $A_{3} A_{2}$ and $S_{3} S_{2}$ are parallel. Changing the indices while keeping the cycling ordering we obtain the equivalent results for the other pairs of lines.)

By definition we have $A_{1} A_{2} \perp w_{\gamma}$ and from Theorem 2.2 we have $\overline{I S_{1}}=\overline{I S_{2}}$. Since $w_{\gamma}$ is interior bisector of $A C$ and $B C$ it also is interior bisector of $n_{I}(A C)$ and $n_{I}(B C)$. Consequently, $S_{1} S_{2} \perp w_{\gamma}$ and thus $S_{1} S_{2}$ is parallel to $A_{1} A_{2}$.

Since $n_{A_{2}}(A B)$ and $n_{A_{1}}(A B)$ are parallel we have $\overline{A_{1} A_{2}}=\overline{S_{1} S_{2}}$. The same is true if we change indices $(1,2,3)$ and $(A, B, C)$, respectively, while keeping the cyclic ordering.

So far we have shown that $\Delta_{A}$ is congruent to $\Delta_{S}$. Now we have to prove that there is a rotation $\rho$ with angle $\pi$ and $\rho\left(\Delta_{A}\right)=\Delta_{S}$.

We observe that $A_{2} S_{2}$ and $A_{3} S_{3}$ are the diagonals of the parallelogram $\Pi_{1}:=\left\{S_{2}, S_{3}, A_{2}\right.$, $\left.A_{3}\right\}$. Thus they intersect in a point $X$. Each of the parallelograms $\Pi_{2}:=\left\{S_{1}, S_{3}, A_{1}, A_{3}\right\}$ and $\Pi_{3}:=\left\{S_{1}, S_{2}, A_{1}, A_{2}\right\}$ shares a diagonal with $\Pi_{1}$. Therefore, the diagonals of $\Pi_{1}$, 
$\Pi_{2}$ and $\Pi_{3}$, respectively, are concurrent in $X$. Consequently, there exists a unique reflection about $X$ which maps $\Delta_{A}$ to $\Delta_{S}$. The existence of this reflection is equivalent to the existence of a rotation $\rho$ about $X$ with angle $\pi$ transforming $\Delta_{A}$ into $\Delta_{S}$.

At last we have to show that $X$ is the FeUERBACH point $F_{\Delta_{S}}$ of $\Delta_{S}$. The base triangle $\Delta$ is the pedal triangle of $\Delta_{A}$. Thus the circumcircle of $\Delta$ is the FEUERBACH circle of $\Delta_{A}$. Since $\rho$ maps $\Delta_{A}$ to $\Delta_{S}$ it maps the corresponding pedal triangles onto each other by reflecting them about $X$. Thus the FEUERBACH circles of $\Delta_{S}$ and $\Delta_{A}$ coincide such as their centers coincide in $X$.

Proof of Theorem 2.4. There is nothing to be done. This theorem is a consequence of the proof of Theorem 2.3 .

Proof of Theorem 2.5. The incenter $I$ of $\Delta$ is the circumcenter of $\Delta_{S}$, see Theorem 2.1. Thus it is contained in the EULER line $e_{\Delta_{S}}$ of $\Delta_{S}$.

Since $e_{\Delta_{S}}$ passes through the FEUERBACH point $F_{\Delta_{S}}$, the rotation $\rho$ with center $F_{\Delta_{S}}$ transforms $e_{\Delta_{S}}$ into $e_{\Delta_{A}}$.

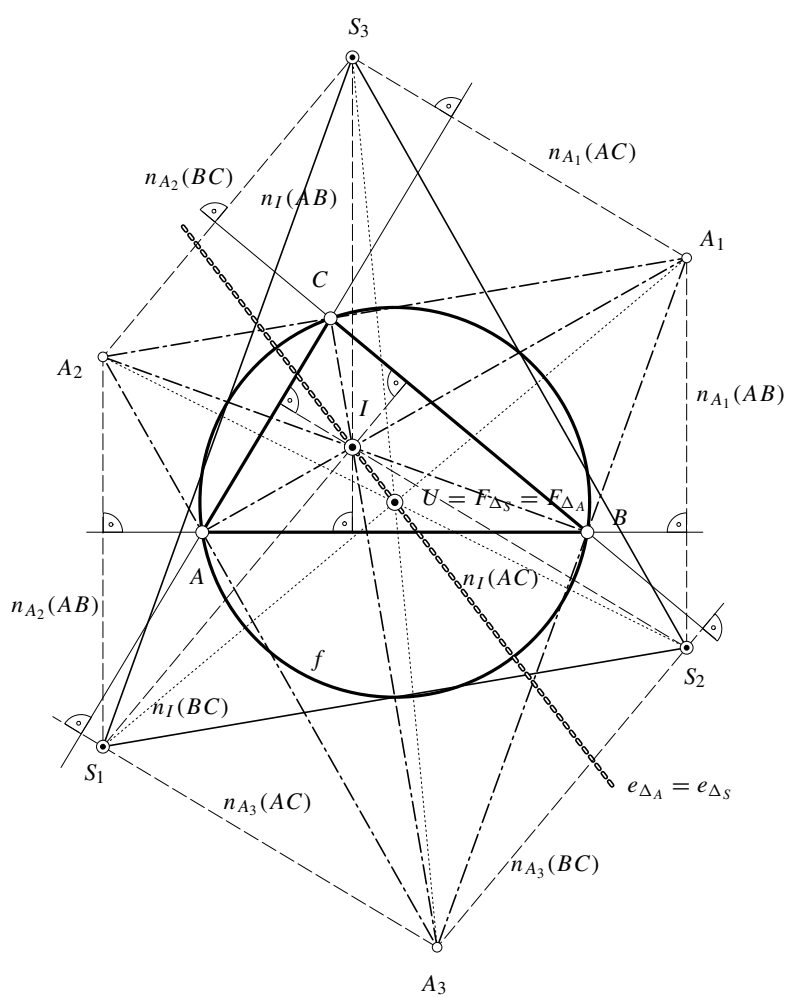

Fig. 4 Triangles $\Delta_{A}$ and $\Delta_{S}$ with common EuLER line $e_{\Delta_{A}}=e_{\Delta_{S}}$ and FEUERBACH circle $f$ 


\section{Alternative interpretation}

As remarked in Section 1, the theorems given in Section 2 can be seen in a different light.

For a given triangle $\Delta_{A}:=\left\{A_{1}, A_{2}, A_{3}\right\}$ draw the orthoptic triangle $\Delta_{O}:=\left\{B_{1}, B_{2}, B_{3}\right\}$, where $B_{i} \in A_{j} A_{k}$ with cyclic ordering of $(i, j, k)$. The orthocenter $H$ of $\Delta_{A}$ is the incenter of $\Delta_{O}$. Now we recall that $\Delta_{A}$ is the excenter triangle of $\Delta_{O}$.

Thus Theorem 2.1 can be reformulated:

Theorem 4.1 (Equivalent to Theorem 2.1)

The normals from the vertex $A_{1}$ of the base triangle $\Delta_{A}$ to the side $B_{1} B_{2}$ of the orthoptic triangle $\Delta_{O}$, the normal from $A_{3}$ to $B_{2} B_{3}$, and the normal through the orthocenter $H$ of $\Delta_{A}$ to $B_{1} B_{3}$ are concurrent in a point $S_{2}$.

This remains true if we change the indices while keeping the cyclic ordering.

Acknowledgements. I have to express my sincere thanks to the reviewer for his hints and comments.

\section{References}

[1] Coxeter, H.S.M.: Introduction to Geometry. J. Wiley \& Sons, New York-London 1961.

[2] Kimberling, C.: Encyclopedia of Triangle Centers. Available at http: //faculty. evansville.edu/ck6/encyclopedia/ETC.html

[3] Schwarz, H.A.: Gesammelte mathematische Abhandlungen. Springer, Berlin 1890.

[4] Steiner, J.: Jacob Steiner's Gesammelte Werke. (2 Bände) Reimer, Berlin 1881.

Boris Odehnal

Institut für Geometrie

Technische Universität Wien

Wiedner Hauptstr. 8-10/113

A-1040 Wien, Österreich

e-mail: boris@geometrie.tuwien.ac.at 